\title{
Surgical Outcome of Cubital Tunnel Syndrome Using the MHQ and DASH: A Retrospective Patient-Reported Assessment
}

\section{Sorg $\mathrm{H}^{*}$, Krämer $\mathrm{R}^{\star 2}$, Reinke $\mathrm{JM}^{3}$ and Knobloch $\mathrm{K}^{4}$}

${ }^{1}$ Department for Plastic, Reconstructive and Aesthetic Surgery, Hand Surgery, Alfried Krupp Krankenhaus, Essen, Germany

${ }^{2}$ Department of Plastic Surgery, Hand Surgery, Burn Unit, University Hospital Schleswig-Holstein

Campus Lübeck, University of Lübeck, Germany

${ }^{3}$ Department of Plastic, Hand and Reconstructive Surgery, Hannover Medical School, Hannover, Germany

${ }^{4}$ Sportpraxis Prof. Dr. med. Karsten Knobloch, Hannover, Germany

1,2 - Authors contributed equally

${ }^{*}$ Corresponding author: Sorg H, M.D., MHBA, Department for Plastic, Reconstructive and Aesthetic Surgery, Hand Surgery, Alfried Krupp Krankenhaus, Hellweg 100, 45276 Essen, Germany, Fax: +49-201805-1172, Tel: +49-201-805-1170, E-mail: heiko.sorg@krupp-krankenhaus.de

Citation: Sorg H, Krämer R, Reinke JM, Knobloch K (2015) Surgical Outcome of Cubital Tunnel Syndrome Using the MHQ and DASH: A Retrospective Patient-Reported Assessment. J Surg Oper Care 1(1): 103.

doi: $10.15744 / 2455-7617.1 .103$

Received Date: March 11, 2015 Accepted Date: August 31, 2015 Published Date: September 02, 2015

\begin{abstract}
Background: Evaluation of the quality of life of adult patients suffering from cubital tunnel syndrome (CUTS), who underwent surgical intervention.

Material and Methods: This retrospective study included hospital chart review and patient-reported outcome assessment using the German version of the Disabilities of the Arm, Shoulder and Hand outcome measure (DASH) and the validated German Michigan Hand Questionnaire (GMHQ).

Results: A total of 41 patients underwent surgery of the cubital tunnel with subcutaneous or submuscular transposition of the ulnar nerve. Mean postoperative GMHQ was $71 \pm 6$. Mean postoperative DASH score was $43 \pm 7$. Satisfaction with surgery was rated positive in $53 \%$, but $89 \%$ would undergo the procedure again. The results of the questionnaire showed great differences between submuscular (GMHQ: 53 \pm 7 DASH: 53 \pm 9 ) and subcutaneous transposition (GMHQ: 69 \pm 7 ; DASH: 34 \pm 10 ) of the ulnar nerve. Better all over results were achieved in male patients compared to women.

Conclusion: The surgical procedure in the treatment of CUTS is associated with an only moderately improved functional benefit and postoperative quality of life in comparison to other arm-/hand-associated diseases. Furthermore, there is still no clear evidence for the superiority of a specific surgical technique.
\end{abstract}

Keywords: DASH; German MHQ; Submuscular transposition; CUTS

\section{Introduction}

In German speaking countries the cubital tunnel syndrome (CUTS) is better known as sulcus nervi ulnaris syndrome. Next to the carpal tunnel syndrome, it is the second most common neuropathic compression disease in the upper limb [1]. Due to the anatomical positioning of the ulnar nerve at the dorsal area of the elbow, it is located in a vulnerable area where it is exposed to stretch and compression forces in the ulnar nerve sulcus. If these lead to constrictions, the patient suffers from paresthesia and reduced sensibility, which aggravate with elbow flexion. Chronic neuropathy is associated with increasing muscle weakness and muscle atrophy of the intrinsic muscles in the hand especially seen in the $4^{\text {th }}$ and $5^{\text {th }}$ finger. In the beginning the CUTS involves numbness and tingling usually on the ulnar side of the hand on palm and back. In further stages of the disease, clawing of fingers four and five might occur.

CUTS can therefore be diagnosed with a differentiated neurological and functional examination, which will be completed with an electrodiagnostic testing. Ultrasound [2] and MRI [3,4] are becoming more important as these techniques allow a direct confirmation of nerve compression or other possible pathologic findings in this area.

Most cases of CUTS are associated with male sex, which might be due to a more prominent tuberculum of the coronoid processus and a thicker fatty pad over the elbow compared to women [5]. The incidence of CUTS is increased in obese patients and in patients who perform repetitive work [6] or hold a tool in position [7]. Contrary, there seems to be no association between the onset of CUTS and the use of vibrating tools or the exposure to external forces [7]. Recently, this disease can also occur with excessive cell phone use and is therefore also called cell phone elbow [8]. 
Acute and subacute ulnar nerve neuropathy will be treated conservatively with the support of splint immobilization [9], avoidance of further external nerve compression and in some cases with repetitive corticosteroid injections [10-12]. If there is no pain relief achievable, surgical intervention is necessary $[11,13,14]$. There are different methods to perform surgery. First of all a simple decompression of the nerve can be done, alternatively subcutaneous, intramuscular or submuscular transposition and medial epicondylectomy are possible [14,15]. Although these methods have been commonly used for approximately 50 years [16], there is no evidence-based recommendation for the most effective surgical treatment option $[11,14,15]$ as the results of comparative studies have been equivocal [14]. However, there are only a few longterm studies using well-established questionnaires [17-19] such as the DASH or the McGowen score (uncommon in Germany), allowing a reliable comparison between the different surgical techniques. Thus it appears that there is ongoing controversy about the optimal therapy option for the patient. This study is the first, which assesses the outcome of CUTS patients after surgery with two accepted and reliable self-report questionnaires (German Michigan Hand Questionnaire (GMHQ); Disabilities of the arm, shoulder and hand (DASH)) in order to clarify the above-mentioned problem.

\section{Materials and Methods}

The study design was a retrospective study that included hospital chart review and patient-reported outcome assessment using the validated German Michigan Hand Questionnaire (GMHQ; [20]) and the Disabilities of the Arm, Shoulder and Hand outcome measure (DASH). A self-created questionnaire has additionally been used to evaluate the postoperative satisfaction, the numeric rating scale (NRS) for pain and preceded corticosteroid therapy. Subjects were identified through a computerized search by ICD10 code for all patients undergoing surgery for CUTS at the Hannover Medical School between January 1, 2006, and December 31, 2010. All patients had been evaluated preoperatively due to the following scheme: history of complaints, physical examination with special focus on neurologic pathologies, $x$-ray of the elbow to exclude osseous pathologies and analysis of nerve conduction velocity by a neurologist. Three out of the following five case definitions had to be met for a patient to undergo surgery: (1) a documented history of pain or neuropathy in the elbow, lower arm or hand for more than one year, (2) ineffective conservative therapy (corticosteroid injection, splinting, analgesia, avoidance of pressure to the cubital tunnel), (3) a documented positive Hoffman-Tinel sign at the cubital tunnel in the patient's medical record, (4) a documented positive Froment's sign in the patient's medical record or (5) reduction of nerve conduction velocity specific for the ulnar nerve. For the inclusion in this study the patients had to suffer from a documented history of pain or neuropathy in the elbow, lower arm and hand in the medical record and had to have a documented surgical intervention for CUTS with submuscular or subcutaneous transposition.

\section{Chart review}

Subjects were identified through a computerized search by ICD-10 code (G56.2) for all patients undergoing surgery for CUTS at our department between January 1,2006, and December 31, 2010. Data collected included age, gender, status of surgeon (resident/ attending), as well as postoperative symptoms and complications. Chart review was done to collect information on age, gender, prior steroid therapy and postoperative symptoms or complications. Forty-one patients were interviewed via GMHQ and DASH, which were delivered to the patients by regular mail.

\section{Outcome measure tools - DASH and German MHQ}

The disabilities of the arm, shoulder and hand outcome measure (DASH) is a data collection instrument composed of 30 questions that ascertain the condition of the upper extremity. On a five-point Likert scale patients rate their ability to perform different daily physical activities using the arm, shoulder or hand (21 items), the severity of pain at rest and activity-related pain, tingling, weakness and stiffness ( 5 items) as well as the impact of pain on social activities, work, sleep, and self-image (4 items). The raw score is then transformed to a score from 0 (no disability) to 100 (most severe disability). The German version of the DASH questionnaire has previously been standardized and validated [21-23].

The Michigan Hand Outcomes Questionnaire (MHQ) has also been developed as a hand specific outcomes tool, applying psychometric principles to create an instrument measuring the health status of patients with hand or wrist disorders. The questionnaire consists of 37 core questions with 5-point scales evaluating the overall hand function, activities of daily living, amount of pain, work performance, aesthetic appearance and patient's satisfaction with the overall hand function. The raw scale score is the sum of responses to each scale item, which is then converted to a score ranging from 0 (worst hand performance) to 100 (best hand performance). The German version of the MHQ questionnaire (MHQ) has been standardized and validated by our own group [20].

The DASH and the German MHQ have been accompanied with further questions about postoperative satisfaction, numeric rating scale (NRS) for pain evaluation and potential preceded corticosteroid therapy. The primary endpoints of this study were defined as the scoring values of the DASH and German MHQ questionnaires.

\section{Surgical procedure}

Under general anesthesia the patient was placed in a supine position with the affected arm supported by a hand table. All operations were performed under Esmarch ischemia with tourniquet control. An approximately 6-8cm long curvilinear longitudinal skin incision was made around the medial epicondyle. The subcutaneous tissue was carefully dissected and the ulnar nerve was ident- 
ified in its sulcus. In case of subcutaneous transposition the ulnar nerve was placed below the subcutaneous fat of the arm and forearm, compared to that the ulnar nerve was placed in the interval between the two heads of flexor carpi ulnaris beneath the flexor pronator origin in the submuscular group. The Osborne ligament was identified and transected. The fascia over the ulnar nerve was divided, and the nerve was deroofed. Care was taken to release the nerve from the intermuscular septum proximally and to the deep flexor aponeurosis distally. The septum within the flexor carpi ulnaris muscle was gently separated if necessary. Meticulous hemostasis was achieved before wound closure.

\section{Results}

\section{Demographic data}

All demographic data and characteristics of the participants and the nonparticipants are presented in Table 1 . A total of 41 patients received surgery between 2006 and 2010 and were invited to participate, 22 patients did not respond or were unwilling to participate after receiving the questionnaire for the assessment. Therefore the data assessed by the DASH or the GMHQ refers to a study population of $n=19$ patients. Data which could be gained from patient chart review refers to $n=41$ patients. The study sample contained 19 women and 22 men. The mean age was $50 \pm 2$ years (range, 23-77 years). The mean follow-up time point after surgery was $38.1 \pm 14.5$ months. At the time of the follow-up examination 18 patients $(95 \%)$ were still working in the same job and 1 patient (5\%) had changed jobs.

\begin{tabular}{|l|l|l|l|}
\hline Independent variables & No. of patients (\%) & GMHQ & DASH \\
\hline Age & & & \\
\hline$<20$ & $0(0 \%)$ & -- & -- \\
\hline $21-30$ & $1(2 \%)$ & n.d. & n.d. \\
\hline $31-40$ & $4(10 \%)$ & n.d. & n.d. \\
\hline $41-50$ & $14(34 \%)$ & $74.8 \pm 7.4$ & $44.7 \pm 10.2$ \\
\hline $51-60$ & $13(32 \%)$ & $71.6 \pm 10.0$ & $36.1 \pm 12.8$ \\
\hline $60+$ & $9(22 \%)$ & $57.0 \pm 22.0$ & $53.3 \pm 11.6$ \\
\hline Gender & & & \\
\hline female & $19(46 \%)$ & $64.4 \pm 8.9$ & $48.4 \pm 10.4$ \\
\hline male & $22(54 \%)$ & $75.5 \pm 7.9$ & $38.9 \pm 9.2$ \\
\hline
\end{tabular}

Table 1: Results of independent variables divided by GMHQ and DASH scores

\section{Patient characteristics}

Perioperative patient characteristics are summed up in Table 2. $90 \%$ of the patients reported complaints about the diseased arm before surgery. These complaints where associated with pain in $74 \%$. The average overall pain level was $4 \pm 1$ at rest and $5 \pm 1$ during stress of the respective arm/elbow. Furthermore patients had difficulties with loss of strength (68\%) in the respective arm and suffered from tingling or loss of sensibility $(84 \%)$. Mean operating time was $93 \pm 13 \mathrm{~min}$. Patient satisfaction with the operative procedure was rated positive in 53\%, but $89 \%$ would undergo the procedure again. Twenty-one percent of the patients had further surgery at the respective arm. Seven out of 19 patients (37\%) reported complications after surgery. These have been scarring $(\mathrm{n}=1)$, reduced sensibility $(n=4)$, pain in the wound area $(n=4)$, hematoma $(n=1)$, perfusion problems $(n=2)$ and problems with arm/ hand function $(\mathrm{n}=2)$.

\section{Postoperative GMHQ and DASH score}

Mean MHQ score was 70.8 \pm 5.9 (95\% CI 74.8-66.8; Figure 1). GMHQ subscores were GMHQ work with 49.5 \pm 7.6 (94\% CI 54.6-

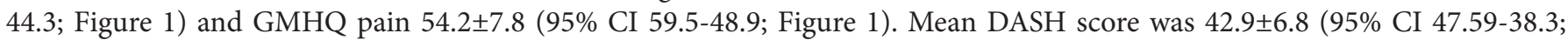
Figure 2), subscores of DASH were DASH work with 51.2 \pm 10.9 (95\% CI 57.0-45.3; Figure 2) and DASH sport was 46.8 \pm 18.1 (95\% CI 53.7-40.0; Figure 2). The GMHQ as well as the DASH are reliable and commonly used questionnaires, which was obvious in this study since they showed a good correlation. However, there are marked differences in the subscores evaluating work items (Figure 3).

\begin{tabular}{|l|l|l|}
\hline Parameter & No. of patients & $\%$ \\
\hline preoperative corticosteroid therapy & 4 & 21 \\
\hline preoperative splinting & 63 & 26 \\
\hline submuscular transposition & 23 & 56 \\
\hline subcutaneous transposition & 18 & 44 \\
\hline general anesthesia & 33 & 80 \\
\hline brachial plexus narcosis & 8 & 20 \\
\hline outpatient surgery & 2 & 2 \\
\hline in hospital surgery & 39 & 98 \\
\hline
\end{tabular}


The questionnaire results differ greatly comparing submuscular and subcutaneous shifting of the ulnar nerve. Whereas the DASH score after submuscular shifting revealed 52.5 \pm 9.1 it was markedly better after subcutaneous shifting with $34.2 \pm 9.6$. For GMHQ the results are $53.3 \pm 6.8$ and $68.7 \pm 7.3$.

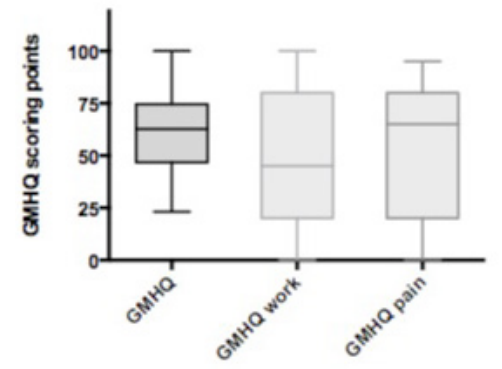

Figure 1: Box plot diagram of the mean total scores for the German MHQ and German MHQ work and pain of adult patients after surgical intervention for cubital tunnel syndrome.

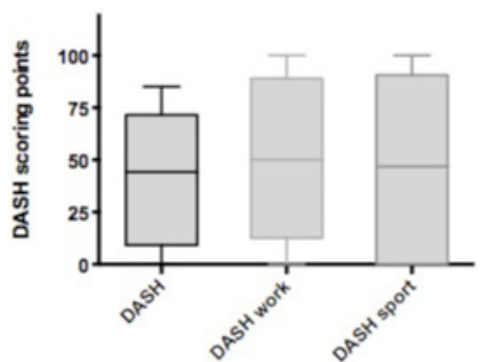

Figure 2: Box plot diagram of the mean total scores for the total DASH and the DASH subscores DASH work and DASH sport/music of adult patients after surgical intervention for cubital tunnel syndrome.

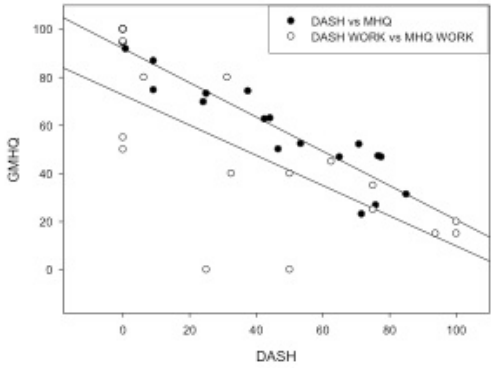

Figure 3: Regression analysis of the results of the DASH and German MHQ as well as of the results of the DASH work and the German MHQ work.

\section{Discussion}

This study revealed the following major findings: Patients suffering of CUTS reveal worse postsurgical outcome scores of GMHQ and DASH in comparison to other hand or arm related diseases [24]. Furthermore, the longterm results of the here presented study are significantly worse than the data that has recently been published by Fitzgerald [18]. The data presented by Fitzgerald included only young and active duty military personnel [18]. In our study, however, elderly patients ( $>60$ years) presented the worst results in both questionnaires. There was no statistical difference between male or female patients, however, marked better results were achieved in male patients compared to women. Interestingly, the questionnaire results demonstrated a major difference between the way of nerve transposition with better results after subcutaneous than submuscular positioning. Preoperative therapy options like steroid treatment or splinting had no effect on the reduction of specific CUTS-symptoms. Only half of the patients were satisfied with the operative procedure, nonetheless nearly $90 \%$ would undergo this procedure again underscoring patientreported outcome measures as crucial key determinant of patient satisfaction [25]. In contrast, Macadam and co-workers analyzed 42 studies in a systematic review of the literature, focusing on the identification and analysis of outcome measures for CUTS, concluding that there are no reliable, reproducible and valid outcome measures for ulnar neuropathy [26]. Furthermore, a current article dealing with patient-related outcome measurements in plastic surgery describes, that especially this form of analysis allows the quantification of the way patients perceive their health and the impact of the treatment on their quality of life [40]. However, as most of such questionnaires are limited by their development, validation or content, the here used hand questionnaires are reliable and validated forms to evaluate hand disease related outcomes [27-29] and might also be used for the evaluation of CUTS-patients [18]. The DASH as well as the GMHQ are reliable questionnaires to evaluate post-operative outcomes as can be seen in nearly equal results (Figure 3), while the subscores assessing respective work items do not correlate well (Figure 3). In this respect, the nonrandomized study design might also be associated with a lower evidence level, however, is more feasible and sufficiently strong enough to draw a respective conclusion [30]. 
For the surgical treatment of CUTS several surgical techniques exist, which have not proven to be superior one over another. Therefore, the surgical treatment of choice is the main topic of the controversial discussion: Although the results between submuscular and subcutaneous shifting of the nerve do not highlight a statistically significant difference, the outcomes of subcutaneous transposition seemed to be better in the here presented evaluation. These results are to some extent in contrast to what is already known and published, as many studies on subcutaneous transposition report worse outcomes with less rates of reduction of symptoms [31-33]. Furthermore, the poor results after subcutaneous transposition might be related to secondary compression by scarring [31]. New prospective randomized studies show no difference between simple nerve decompression and subcutaneous [34-36] or submuscular transposition [37,38], although transposition surgery was associated with more complications than simple decompression [34]. In a decision analysis comparing the four most common surgical techniques for CUTS (simple decompression of the cubital tunnel, medial epicondylectomy, anterior subcutaneous transposition and anterior submuscular transposition), Brauer and Graham concluded that the decompression and subcutaneous transposition showed the highest expected utility and should therefore remain the preferred strategies in CUTS treatment [39]. As also shown in our study, conservative treatment with corticosteroid-injection or splinting prior to surgery did not reveal any benefit. This is in line with reports on the non-operative treatment, which were associated with low benefit and high recurrence rates. In these cases neuropathic symptoms could not be reduced by changes in the daily use of the hand, since the damage done to the ulnar nerve was already irreversible only due to already seriously injured ulnar nerves [31].

The GMHQ and DASH questionnaire as patient-related outcome measures for the evaluation of the surgical results of CUTS represent a convenient and reliable tool. Patient's satisfaction after surgery was good, although they did not have excellent results compared to other arm/hand-related disease treatments. Furthermore, there is still no clear evidence for the superiority of one surgical method, providing a basis for ongoing controversial discussion on that topic.

\section{References}

1. Elhassan B, Steinmann SP (2007) Entrapment neuropathy of the ulnar nerve. J Am Acad Orthop Surg 15: 672-81.

2. Wiesler ER, Chloros GD, Cartwright MS, Shin HW, Walker FO (2006) Ultrasound in the diagnosis of ulnar neuropathy at the cubital tunnel. J Hand Surg Am 31: 1088-93.

3. Britz GW, Haynor DR, Kuntz C, Goodkin R, Gitter A, et al. (1996) Ulnar nerve entrapment at the elbow: correlation of magnetic resonance imaging, clinical, electrodiagnostic, and intraoperative findings. Neurosurgery 38: 458-65.

4. Vucic S, Cordato DJ, Yiannikas C, Schwartz RS, Shnier RC (2006) Utility of magnetic resonance imaging in diagnosing ulnar neuropathy at the elbow. Clin Neurophysiol 117: 590-5.

5. Contreras MG, Warner MA, Charboneau WJ, Cahill DR (1998) Anatomy of the ulnar nerve at the elbow: potential relationship of acute ulnar neuropathy to gender differences. Clin Anat 11:372-8.

6. Descatha A, Leclerc A, Chastang JF, Roquelaure Y, Study Group on Repetitive Work (2004) Incidence of ulnar nerve entrapment at the elbow in repetitive work. Scand J Work Environ Health 30: 234-40.

7. van Rijn RM, Huisstede BM, Koes BW, Burdorf A (2009) Associations between work-related factors and specific disorders at the elbow: a systematic literature review. Rheumatology (Oxford) 48: 528-36.

8. Darowish M, Lawton JN, Evans PJ (2009) Q:What is cell phone elbow, and what should we tell our patients? Cleve Clin J Med 76: $306-8$.

9. Szabo RM, Kwak C (2007) Natural history and conservative management of cubital tunnel syndrome. Hand Clin 23: 311-8.

10. Dellon AL, Hament W, Gittelshon A (1993) Nonoperative management of cubital tunnel syndrome: an 8-year prospective study. Neurology 43: 1673-7.

11. Assmus H, Antoniadis G, Bischoff C, Hoffmann R, Martini AK, et al. (2009) Diagnosis and therapy of cubital tunnel syndrome--state of the art. Handchir Mikrochir Plast Chir 41: 2-12.

12. Hong CZ1, Long HA, Kanakamedala RV, Chang YM, Yates L (1996) Splinting and local steroid injection for the treatment of ulnar neuropathy at the elbow: clinical and electrophysiological evaluation. Arch Phys Med Rehabil 77: 573-7.

13. Robertson C, Saratsiotis J (2005) A review of compressive ulnar neuropathy at the elbow. J Manipulative Physiol Ther 28: 345.

14. Palmer BA, Hughes TB (2010) Cubital tunnel syndrome. J Hand Surg Am 35: 153-63.

15. Bartels RH, Menovsky T, Van Overbeeke JJ, Verhagen WI (1998) Surgical management of ulnar nerve compression at the elbow: an analysis of the literature. J Neurosurg 89: 722-7.

16. Bartels RH (2001) History of the surgical treatment of ulnar nerve compression at the elbow. Neurosurgery 49: $391-9$.

17. Mitsionis GI, Manoudis GN, Paschos NK, Korompilias AV, Beris AE (2010) Comparative study of surgical treatment of ulnar nerve compression at the elbow. J Shoulder Elbow Surg 19: 513-9.

18. Fitzgerald BT, Dao KD, Shin AY (2004) Functional outcomes in young, active duty, military personnel after submuscular ulnar nerve transposition. J Hand Surg 29: 619-24.

19. Schnabl SM, Kisslinger F, Schramm A, Dragu A, Kneser U, et al. (2011) Subjective outcome, neurophysiological investigations, postoperative complications and recurrence rate of partial medial epicondylectomy in cubital tunnel syndrome. Arch Orthop Trauma Surg 131: 1027-33.

20. Knobloch K, Kuehn M, Papst S, Kraemer R, Vogt PM (2011) German standardized translation of the michigan hand outcomes questionnaire for patient-related outcome measurement in Dupuytren disease. Plast Reconstr Surg 128: 39-40e.

21. Germann G, Wind G, Harth A (1999) [The DASH(Disability of Arm-Shoulder-Hand) Questionnaire--a new instrument for evaluating upper extremity treatment outcome]. Handchir Mikrochir Plast Chir 31: 149-52.

22. Germann G, Harth A, Wind G, Demir E (2003) Standardisierung und Validierung der deutschen Version 2.0 des "Disability of Arm, Shoulder, Hand" (DASH)Fragebogens zur Outcome-Messung an der oberen Extremität. Der Unfallchirurg 106: 13-9.

23. Offenbächer M, Ewert T, Sangha O, Stucki G (2003) Validation of a German version of the "Disabilities of Arm, Shoulder and Hand" questionnaire (DASH-G). Z Rheumatol 62: 168-77. 
24. Niekel MC, Lindenhovius AL, Watson JB, Vranceanu AM, Ring D (2009) Correlation of DASH and QuickDASH with measures of psychological distress. J Hand Surg Am 34: 1499-505.

25. Carr-Hill RA (1992) The measurement of patient satisfaction. J Public Health Med 14: 236-49.

26. Macadam SA, Bezuhly M, Lefaivre KA (2009) Outcomes measures used to assess results after surgery for cubital tunnel syndrome: a systematic review of the literature. J Hand Surg Am 34: 1482-91.

27. Levine DW, Simmons BP, Koris MJ, Daltroy LH, Hohl GG, et al. (1993) A self-administered questionnaire for the assessment of severity of symptoms and functional status in carpal tunnel syndrome. J Bone Joint Surg Am 75: 1585-92.

28. Dias JJ, Rajan RA, Thompson JR (2008) Which questionnaire is best? The reliability, validity and ease of use of the Patient Evaluation Measure, the Disabilities of the Arm, Shoulder and Hand and the Michigan Hand Outcome Measure. J Hand Surg Eur 33: 9-17.

29. Zyluk A, Piotuch B (2011) A Comparison of DASH, PEM and Levine questionnaires in outcome measurement of carpal tunnel release. Handchir Mikrochir Plast Chir 43: 162-6.

30. Graham B (2005) Strategies for nonrandomized clinical research in hand surgery. Clin Plast Surg 32: 529-36.

31. Mowlavi A, Andrews K, Lille S, Verhulst S, Zook EG, et al. (2000) The management of cubital tunnel syndrome: a meta-analysis of clinical studies. Plast Reconstr Surg 106: 327-34.

32. Nikitins MD, Griffin PA, Ch'ng S, Rice NJ (2002) A dynamic anatomical study of ulnar nerve motion after anterior transposition for cubital tunnel syndrome. Hand Surg 7: 177-82.

33. Nouhan R, Kleinert JM (1997) Ulnar nerve decompression by transposing the nerve and z-lengthening the flexor-pronator mass: clinical outcome. J Hand Surg Am 22: 127-31.

34. Bartels RH, Verhagen WI, van der Wilt GJ, Meulstee J, van Rossum LG, et al. (2005) Prospective randomized controlled study comparing simple decompression versus anterior subcutaneous transposition for idiopathic neuropathy of the ulnar nerve at the elbow: Part 1. Neurosurgery 56: 522-30.

35. Nabhan A, Ahlhelm F, Kelm J, Reith W, Schwerdtfeger K, et al. (2005) Simple decompression or subcutaneous anterior transposition of the ulnar nerve for cubital tunnel syndrome. J Hand Surg Br 30: 521-4.

36. Nabhan A, Kelm J, Steudel WI, Shariat K, Sova L, et al. (2007) Cubital tunnel syndrome - simple nerve decompression or decompression with subcutaneous anterior transposition? Fortschr Neurol Psychiatr 75: 168-71.

37. Gervasio O, Gambardella G, Zaccone C, Branca D (2005) Simple decompression versus anterior submuscular transposition of the ulnar nerve in severe cubital tunnel syndrome: a prospective randomized study. Neurosurgery 56: 108-17.

38. Biggs M, Curtis JA (2006) Randomized, prospective study comparing ulnar neurolysis in situ with submuscular transposition. Neurosurgery 58: 296-304.

39. Brauer CA, Graham B (2007) The surgical treatment of cubital tunnel syndrome: a decision analysis. J Hand Surg Eur 32: 654-62.

40. Pusic AL, Lemaine V, Klassen AF, Scott AM, Cano SJ (2011) Patient-reported outcome measures in plastic surgery: Use and interpretation in evidence-based medicine. Plast Reconstr Surg 127: 1361-7.

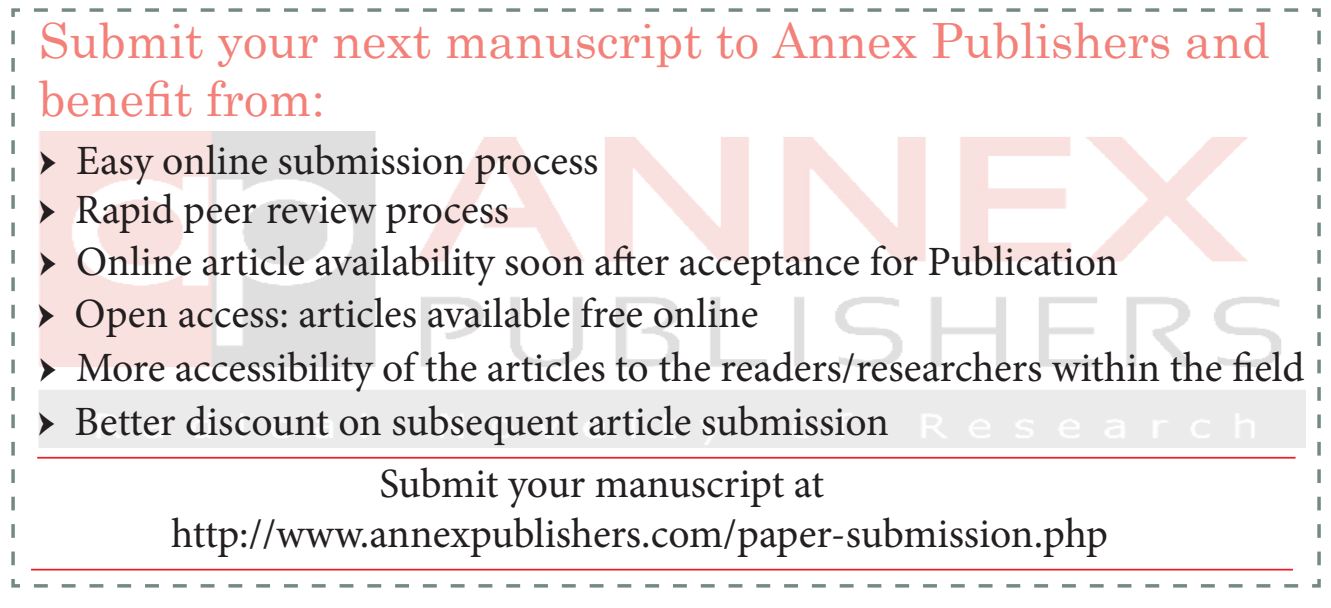

